\title{
Caracterização físico-química e sensorial de sucos da uva Isabel em cortes com diferentes variedades produzidas na região do Vale do Rio do Peixe-SC
}

\author{
Physicalchemical and sensorial characterization of 'Isabel' \\ grape juices in cuts with different varieties produced in the \\ Rio do Peixe-SC region-Brazil
}

Angélica Bender ${ }^{1 *}$ (D), André Luiz Kulkamp de Souza², Vinicius Caliari², Marcelo Barbosa Malgarim¹, Vagner Brasil Costa ${ }^{1}$, Carolina Goulart ${ }^{1}$

${ }^{1}$ Universidade Federal de Pelotas (UFPel), Campus Universitário, Capão do Leão/RS - Brasil ${ }^{2}$ Empresa de Pesquisa e Extensão Rural de Santa Catarina (EPAGRI), Estação Experimental de Videira, Videira/SC - Brasil

*Corresponding Author: Angélica Bender, Universidade Federal de Pelotas (UFPel), Campus Universitário, s/n, CEP 96160-000, Capão do Leão/RS - Brasil, e-mail: bender.angelica.fruti@gmail.com

Cite as: Bender, A., Souza, A. L. K., Caliari, V., Malgarim, M. B., Costa, V. B., \& Goulart, C. (2020).

Physicalchemical and sensorial characterization of 'Isabel' grape juices in cuts with different varieties produced in the Rio do Peixe-SC region-Brazil. Brazilian Journal of Food Technology, 23, e2019187.

https://doi.org/10.1590/1981-6723.18719

\begin{abstract}
Resumo
O maior volume de suco de uva produzido no Brasil é da cultivar Isabel. No entanto, é necessário que se façam cortes com outras cultivares para melhorar a qualidade do suco, o que eleva a demanda por outras cultivares, especialmente Bordô. Sendo assim, o objetivo deste estudo foi avaliar a influência das cultivares BRS Rúbea, BRS Cora, BRS Violeta, BRS Carmem, BRS Magna e Bordô em cortes com Isabel quanto às características físico-químicas, colorimétricas e sensoriais de sucos produzidos na Região do Vale do Rio do Peixe - SC. Os sucos foram elaborados na Epagri - Estação Experimental de Videira - SC, sendo: (ISA) 100\% Isabel; (ISA+RUB) 70\% Isabel + 30\% Rúbea: (ISA+COR) 70\% Isabel + 30\% Cora; (ISA+VIO) 70\% Isabel + 30\% Violeta; (ISA+CAR) 70\% Isabel + 30\% Carmem; (ISA+MAG) 70\% Isabel + 30\% Magna; (ISA+BOR) 70\% Isabel + 30\% Bordô, e avaliados quanto à composição físico-química, colorimétrica e perfil sensorial. A maior acidez titulável foi verificada em ISA+MAG, seguida de ISA+BOR. Os sucos ISA+VIO obtiveram a maior média para o teor de sólidos solúveis, não diferindo apenas de ISA+CAR, que também apresentou a menor acidez dentre os tratamentos. Os sucos ISA+VIO e ISA+CAR apresentaram maior teor de sólidos solúveis em relação ao suco de Isabel 100\%. A menor luminosidade foi observada nos sucos do ISA+VIO, seguida por ISA+CAR. Quanto aos atributos sensoriais foram observadas diferenças significativas apenas para cor e acidez. Os sucos ISA+VIO, ISA+CAR e ISA+BOR receberam as maiores notas para o atributo cor. Todas as cultivares testadas influenciaram as características colorimétricas do suco, mostrando-se alternativas para a melhoria da cor de sucos de Isabel. Sensorialmente, apenas a cor e a acidez foram influenciadas pelos diferentes cortes.
\end{abstract}

Palavras-chave: Vitis labrusca; Varietal; Híbridas; Variedades tintórias. 


\begin{abstract}
The highest volume of grape juices from Brazil is produced from Isabel cultivar. However, $t$ cuts from other cultivars are essential for improving quality, which increases the demand for other cultivars, especially the 'Bordô'. The objective of this study was to evaluate the influence of the BRS Rúbea, BRS Cora, BRS Violeta, BRS Carmem, BRS Magna and Bordô cultivars in cuts with 'Isabel', in terms of the physicochemical, colorimetric and sensorial characteristics of juices produced at the Vale do Rio do Peixe-SC region-Brazil. The juices were elaborated at the Epagri- Experimental Station of Videira-SC, from: (ISA) 100\% Isabel; (ISA + RUB) $70 \%$ Isabel + 30\% Rúbea: (ISA + COR) 70\% Isabel + 30\% Cora; (ISA + VIO) 70\% Isabel + 30\% Violet; (ISA + CAR) 70\% Isabel + 30\% Carmem; (ISA + MAG) 70\% Isabel + 30\% Magna; (ISA + BOR) 70\% Isabel + 30\% Bordô. It was evaluated their physicochemical, colorimetric and sensorial profiles. The highest titratable acidity was verified in ISA + MAG juice, followed by ISA + BOR. The ISA + VIO juice obtained the highest average for the soluble solids content, which did not differ only from ISA + CAR and also presented the lowest acidity among the treatments. The ISA + VIO and ISA + CAR juices presented an increase in the soluble solids content in relation to the $100 \%$ Isabel juice. The lowest luminosity was observed in the ISA + VIO juice, followed by ISA + CAR. For the sensorial attributes, significant differences in the different cuts were only observed for color and acidity. The ISA + VIO, ISA + CAR and ISA + BOR juices received the highest scores for the color attribute. All cultivars tested influenced the colorimetric characteristics of the juice, showing alternatives to improve the color of 'Isabel' juices.
\end{abstract}

Keywords: Vitis labrusca; Varietal; Hybrids; Tinting cultivars.

\title{
1 Introdução
}

O suco de uva integral é um derivado não alcoólico da uva que reflete as características de cor, aroma e sabor da fruta que o gerou. Os benefícios para a saúde relacionados com os polifenóis da uva geram um interesse crescente pela qualidade e potencial bioativo do suco de uva, elevando o consumo nos últimos anos, quadruplicando o volume de vendas entre os anos de 2005 a 2015 (Toaldo et al., 2015; Fongaro, et al., 2016; Mota et al., 2018).

A elaboração do suco de uva pode ocorrer a partir de qualquer variedade, desde que tenha atingido a maturação adequada. Em países de tradição vitícola o suco é elaborado com uvas Vitis vinifera (Borges et al., 2011; Bender et al., 2017). No Brasil, os sucos são elaborados com uvas Vitis labrusca, conhecidas como americanas e híbridas e estas representam mais de $80 \%$ da uva processada no país, com destaque para as cultivares Bordô e Isabel, que obtiveram produções próximas a 160 e 262 milhões de quilos na safra 2017, respectivamente (Toaldo et al., 2013, 2015; Mello \& Machado, 2018). No estado de Santa Catarina foram processados mais de 30 milhões de quilos de uva na safra 2018, sendo que aproximadamente 22 milhões foram de Isabel e Bordô (Empresa de Pesquisa e Extensão Rural de Santa Catarina, 2018).

A cultivar Isabel é a responsável pelo maior volume de suco de uva produzido no Brasil, devido à grande disponibilidade de matéria prima. No entanto, normalmente os sucos elaborados com esta uva precisam ser cortados com sucos de cultivares tintureiras para melhoria da qualidade da cor (Camargo et al., 2010). De acordo com Pereira et al. (2008), a escolha de diferentes cultivares para a produção de suco mostra-se favorável, pois possibilita produzir sucos através de misturas, corrigindo eventuais desequilíbrios ocorridos quando produzidos individualmente.

Ferri et al. (2017) alegam que a cultivar Bordô é uma excelente opção para cortes com sucos de Isabel devido ao seu potencial de coloração. No entanto, via de regra, a maturação da Bordô ocorre em períodos chuvosos nos estados do sul e sudeste brasileiro, prejudicando a qualidade das uvas, e por esta razão não atingem uma relação de açúcar e acidez suficiente para elaboração de um produto de qualidade, prejudicando por vezes o enquadramento do suco à legislação vigente (Chiarotti et al., 2011). Outro problema enfrentado em relação ao cultivo de Bordô é a oscilação na produção devido a distúrbios fisiológicos que prejudicam a 
frutificação, reduzindo significativamente a produção em determinadas safras (Miotto et al., 2014; Castilhos et al., 2016).

Visando ampliar a oferta de uvas para processamento de suco, foram lançadas nos últimos anos novas cultivares que se destacam pelos altos teores de açúcar e cor. Dentre elas estão BRS Carmem, BRS Rúbea, BRS Violeta, BRS Cora e BRS Magna. Essas variedades podem ser utilizadas na elaboração de sucos puros ou em cortes com as cultivares tradicionais (Camargo et al., 2010; Borges et al., 2011).

Borges et al. (2011) avaliaram sensorialmente cortes de sucos de Isabel com as variedades Concord, BRS Rúbea, BRS Cora, BRS Violeta e BRS Carmem, no norte do Paraná. Os autores concluíram que as variedades testadas, com exceção da Concord, podem ser usadas em cortes com a Isabel para melhoria da cor. Dutra et al. (2014) avaliaram sucos varietais de cultivares Vitis vinifera e Vitis labrusca, concluindo que a cultivar BRS Cora apresentou maior índice de polifenóis totais e antocianinas totais, podendo ser uma alternativa para a melhoria da cor em cortes de sucos. Mota et al. (2018) avaliaram sucos produzidos no estado de Minas Gerais, observando que sucos varietais de Isabel Precoce apresentaram baixa concentração de compostos fenólicos, mas quando misturados a sucos de BRS Violeta, Concord e Bordô, ocorreu aumento do conteúdo polifenólico.

A cultivar Isabel apresenta predominância nas regiões produtoras no sul do Brasil, em especial na Região do Vale do Rio do Peixe - SC. Porém a necessidade de melhoria da coloração dos sucos produzidos com Isabel amplia a demanda por outras cultivares, em especial a Bordô, que em determinadas safras é insuficiente devido à baixa produtividade, levando as indústrias a buscarem a matéria prima dos estados vizinhos, elevando os custos de produção. Em 2018 foram processadas mais de 8 milhões de quilos de uvas oriundas dos estados do Rio Grande do Sul e Paraná (Empresa de Pesquisa e Extensão Rural de Santa Catarina, 2018),

O emprego de diferentes cultivares aptas à elaboração de suco se mostra uma alternativa para contornar os problemas relacionados à baixa disponibilidade da cultivar Bordô na Região do Vale do Rio do Peixe - SC. No entanto, Borges et al. (2011) alertam que o paladar do consumidor brasileiro está habituado as características gustativas do suco de Isabel, exigindo cautela e testes para avaliar uma mudança na matriz varietal. O Vale do Rio do Peixe - SC é a região mais antiga e tradicional de produção de uva no estado, porém são escassos os trabalhos referentes às diferentes cultivares lançadas nos últimos anos nesta região. Neste contexto, o objetivo deste estudo foi avaliar a influência das cultivares BRS Rúbea, BRS Cora, BRS Violeta, BRS Carmem, BRS Magna e Bordô em cortes com Isabel quanto às características físico-químicas, colorimétricas e sensoriais de sucos produzidos na Região do Vale do Rio do Peixe - SC.

\section{Material e métodos}

Os sucos foram elaborados a partir de uvas colhidas nos meses de janeiro e fevereiro de 2017, na área experimental da Epagri - Empresa de Pesquisa Agropecuária e Extensão Rural de Santa Catarina, Estação Experimental de Videira (Videira, SC/Brasil), localizada sob a coordenada latitude 27 $02^{\prime} 27,59^{\prime \prime}$ S, longitude $51^{\circ} 08^{\prime} 04,73^{\prime \prime} \mathrm{W}$, altitude de 830 metros acima do nível do mar. O clima da região, de acordo com Köppen, é classificado como mesotérmico úmido e verão ameno (Cfb). As médias históricas (1960-2016) de precipitação, temperatura média e umidade relativa nos meses que englobam a brotação e colheita (setembro a março) foram de $1.240 \mathrm{~mm}, 19,8{ }^{\circ} \mathrm{C}$ e $73,4 \% \mathrm{UR}$, respectivamente (EPAGRI/CIRAM). As uvas empregadas na elaboração dos sucos foram: Isabel, BRS Rúbea, BRS Cora; BRS Violeta; BRS Carmem, BRS Magna e Bordô, produzidas em vinhedo experimental, implantado em 2008 no sistema de condução em ípsilon (Y) sob o porta-enxerto VR 043-43 em espaçamento de 3,0 x 2,0 m, entre linhas e entre plantas, respectivamente. $\mathrm{O}$ ponto de colheita das uvas foi determinado de acordo com a evolução da curva de maturação, considerando a acidez total e teor de sólidos solúveis avaliados semanalmente. A partir da data de troca de cor e colheita (Tabela 1) foi efetuada a mediada que as variáveis físico-químicas se mostraram constantes variando de acordo com diferentes variedades (Tabela 2). 
Tabela 1. Subperíodos fenológicos das variedades de uva Isabel, BRS Rúbea, BRS Cora, BRS Violeta, BRS Carmem, BRS Magna e Bordô produzidas na Região do Vale do Rio do Peixe na safra 2017.

\begin{tabular}{cccc}
\hline \multirow{2}{*}{ Tratamentos } & \multicolumn{3}{c}{ Variáveis } \\
\cline { 2 - 4 } & Acidez titulável $\left(\mathbf{m E q} \mathbf{~ L}^{-1}\right)$ & Sólidos Solúveis ( ${ }^{\circ}$ Brix) & Produtividade (ton/ha) \\
\hline ISA & 60,08 & 15,80 & 19,17 \\
\hline RUB & 95,59 & 14,60 & 26,02 \\
\hline COR & 112,47 & 17,30 & 11,05 \\
\hline VIO & 117,15 & 17,90 & 12,06 \\
\hline CAR & 106,48 & 18,50 & 19,96 \\
\hline MAG & 161,21 & 16,00 & 4,80 \\
\hline BOR & 86,18 & 14,80 & 32,05 \\
\hline
\end{tabular}

ISA (Isabel); RUB (BRS Rúbea); COR (BRS Cora); VIO (BRS Violeta); CAR (BRS Carmem); MAG (BRS Magna) e BOR (Bordô).

Tabela 2. Produtividade e aspectos físico-químicos das uvas no momento da colheita das variedades de uva Isabel, BRS Rúbea, BRS Cora, BRS Violeta, BRS Carmem, BRS Magna e Bordô produzidas na Região do Vale do Rio do Peixe na safra 2017.

\begin{tabular}{|c|c|c|c|c|}
\hline \multirow[b]{2}{*}{ Tratamentos } & \multicolumn{4}{|c|}{ Subperíodos fenológicos } \\
\hline & Poda & Mudança de cor & Colheita & $\begin{array}{c}\text { Duração do ciclo } \\
\text { (dias) }\end{array}$ \\
\hline ISA & $31 / 08 / 16$ & $17 / 01 / 17$ & $02 / 03 / 17$ & 183 \\
\hline RUB & $31 / 08 / 16$ & $27 / 12 / 16$ & $21 / 02 / 17$ & 174 \\
\hline COR & $29 / 08 / 16$ & $27 / 12 / 16$ & $21 / 02 / 17$ & 176 \\
\hline $\mathrm{VIO}$ & $29 / 08 / 16$ & $28 / 11 / 16$ & $23 / 01 / 17$ & 147 \\
\hline CAR & $31 / 08 / 16$ & $19 / 01 / 17$ & $02 / 03 / 17$ & 183 \\
\hline MAG & $31 / 08 / 16$ & $05 / 12 / 17$ & $01 / 02 / 17$ & 154 \\
\hline BOR & $31 / 08 / 16$ & $25 / 12 / 17$ & $01 / 02 / 17$ & 154 \\
\hline
\end{tabular}

ISA (Isabel); RUB (BRS Rúbea); COR (BRS Cora); VIO (BRS Violeta); CAR (BRS Carmem); MAG (BRS Magna) e BOR (Bordô).

As amostras de sucos foram elaboradas em duas etapas:

$1^{\circ}$ etapa: elaboração dos sucos varietais no equipamento denominado suquificador integral. O desengace foi realizado de forma mecânica com desengaçadeira modelo DZ-25 e posteriormente as bagas foram levadas ao suquificador integral, construído em aço inoxidável e desenvolvido pela Embrapa Uva e Vinho em 2016. As uvas foram colocadas no tambor perfurado localizado no interior do equipamento, em que se adicionaram enzimas (Pectinex Ultra SP-L) na concentração de $3 \mathrm{~g} \mathrm{hL}^{-1}$. A homogeneização foi feita pelo próprio equipamento. As uvas permaneceram no interior dele por um período de uma hora, à temperatura de $\pm 50^{\circ} \mathrm{C}$. Posteriormente, realizou-se a prensagem para separação do líquido, que foi conduzido para a câmara fria a uma temperatura de $\pm 1{ }^{\circ} \mathrm{C}$ para decantação das partículas sólidas por um período de 24 horas. No dia seguinte, o suco foi trasfegado, pasteurizado e engarrafado a uma temperatura de $86{ }^{\circ} \mathrm{C}$, devidamente etiquetado e armazenado até o momento da realização dos cortes.

$2^{\circ}$ etapa: realização dos cortes, que consistiu na mistura do suco de Isabel com as demais variedades, após a medida do volume necessário de cada suco varietal para a relação de $70 \%$ Isabel $+30 \%$ de cada uma das 
demais variedades testadas. Os cortes foram efetuados adicionando $1,75 \mathrm{~L}$ de suco Isabel e $0,75 \mathrm{~L}$ das diferentes variedades, totalizando 2,5 L de cada tratamento. Após a devida homogeneização dos sucos, esses foram pasteurizados em um sistema adaptado, devido ao pouco volume de suco. Esse sistema consistiu em um recipiente de aço inoxidável sobre um fogareiro, em que os sucos foram constantemente homogeneizados, envazados à temperatura de $86^{\circ} \mathrm{C}$ e armazenados até o momento das análises.

Os tratamentos foram definidos como:

ISA- 100\% Isabel;

ISA+RUB - 70\% Isabel + 30\% Rúbea;

ISA+COR - 70\% Isabel + 30\% Cora;

ISA+VIO - 70\% Isabel + 30\% Violeta;

ISA +CAR - 70\% Isabel + 30\% Carmem;

ISA + MAG - 70\% Isabel + 30\% Magna;

ISA+BOR - 70\% Isabel $+30 \%$ Bordô.

O delineamento experimental utilizado foi inteiramente casualizado, arranjado em esquema unifatorial, com três repetições. O fator de tratamento testou diferentes cortes (ISA, ISA+RUB, ISA+COR, ISA+VIO, ISA + CAR, ISA+MAG e ISA+BOR). Foram utilizadas 5 garrafas de vidro com capacidade de $500 \mathrm{~mL}$ cada para cada tratamento, sendo três escolhidas aleatoriamente para as análises físico-químicas, colorimétricas e compostos bioativos. Cada garrafa representou uma repetição. O restante das amostras foi destinado à análise sensorial.

O teor de sólidos solúveis foi determinado em refratômetro digital de bancada com compensação automática de temperatura (QUIMIS ${ }^{\circledR}$ ), e o resultado expresso em ${ }^{\circ}$ Brix. $\mathrm{O}$ pH foi determinado em pHmetro Meter $\mathrm{AD} 1030$ e as determinações de acidez total foram realizadas por titulação da amostra, com solução padronizada de $\mathrm{NaOH} 0,1 \mathrm{~N}$, adotando-se como ponto final da titulação o $\mathrm{pH}=8,2$ e o resultado expresso em $\mathrm{mEq} \mathrm{L}^{-1}$ (International Organisation of Vine and Wine, 2009). A relação entre ambos foi determinada pela obtenção do quociente da divisão entre os sólidos solúveis ( ${ }^{\circ}$ Brix) e acidez titulável.

As colorações dos sucos foram determinadas pelo sistema CIE $\mathrm{L}^{*} \mathrm{a}^{*} \mathrm{~b}^{*}$, com o colorímetro modelo Minolta CM-5 e expressa nos parâmetros de cor L*, a* e $b^{*}$. Com esses parâmetros foram calculadas as coordenadas cilíndricas $\mathrm{H}^{\circ}$ e $\mathrm{C}^{*}$, em que $\mathrm{H}^{\circ}$ define a tonalidade (Ângulo Hue) e $\mathrm{C}^{*}$ o croma (McGuire, 1992). O ângulo Hue $\left(\mathrm{H}^{\circ}\right)$ e o croma $\left(\mathrm{C}^{*}\right)$ foram calculados através das equações $\mathrm{H}^{\circ}=\tan ^{-1}\left(\mathrm{~b}^{*} / \mathrm{a}^{*}\right)$ e $\mathrm{C}^{*}=\left[\left(\mathrm{a}^{*}\right)^{2}+\left(\mathrm{b}^{*}\right)^{2}\right]^{1 / 4}$, respectivamente. A diferença total de cor $\left(\Delta \mathrm{E}^{*}\right)$ foi calculada por meio da equação $\Delta \mathrm{E}^{*}=\left[\left(\Delta \mathrm{L}^{*}\right)^{2}+\left(\Delta \mathrm{a}^{*}\right)^{2}+\left(\Delta \mathrm{b}^{*}\right)^{2}\right]$, utilizando o suco $100 \%$ Isabel como padrão, onde o $\Delta$ é a variação entre cada coordenada de cor da amostra padrão (100\% Isabel) e a amostra dos cortes.

A avaliação sensorial dos sucos foi realizada através da análise quantitativa (NBR 12994) (Associação Brasileira De Normas Técnicas, 2014). A intensidade dos atributos foi avaliada em escala não estruturada de 9 pontos, ancorada nos extremos com as palavras pouca intensidade e muita intensidade para cor, desagradável e agradável para aroma, baixa e alta para acidez e doçura, pouco e muito para o equilíbrio doçura/acidez. A compreensão dos atributos e descritores pelos provadores foi verificada e avaliada durante a seleção e treinamento da equipe de degustadores. $O$ treinamento foi realizado durante 6 meses precedentes ao início das avaliações. Foram efetuados testes de gostos (doce, ácido e amargo) com soluções teste e posteriormente familiarização com as amostras e sucos de uva comerciais. Foi selecionado um grupo de 10 pessoas que assinaram o Termo de Consentimento Livre e Esclarecido aprovado pelo Comitê de Ética da Universidade Federal de Pelotas, sob protocolo CAAE 92226218.8.0000.5317. Os avaliadores receberam as amostras $\left(20 \pm 1{ }^{\circ} \mathrm{C}\right)$ em taças de vidro codificadas com três dígitos aleatórios, juntamente com as fichas de avaliação. 
Os dados foram submetidos à análise de variância (ANOVA) e quando detectados efeitos de tratamento, procedeu-se o teste de comparação de médias pelo Teste Tukey a $5 \%$ de probabilidade de erro.

\section{Resultados e discussão}

A Tabela 3 mostra as médias e diferenças significativas ao nível de 5\% entre as amostras de sucos de uva para as variáveis de $\mathrm{pH}$, acidez titulável, sólidos solúveis e relação SS/AT. Os sucos dos tratamentos ISA, ISA+RUB e ISA+COR não apresentaram diferenças significativas entre si para o $\mathrm{pH}$, enquanto os sucos do tratamento ISA + VIO obtiveram maior valor para esta mesma variável, diferindo dos demais. De acordo com Rizzon \& Meneguzzo (2007), o valor mínimo do pH de sucos de uva varia de 3,00 a 3,10, alegando que o pH associado a tratamentos térmicos, como a pasteurização, apresenta influência na inativação de leveduras e bactérias que possam vir a contaminar os sucos, influenciando a temperatura e tempo do processo de estabilização microbiológica, considerando que quanto mais baixo for o valor do $\mathrm{pH}$, mais fácil a destruição térmica dos microrganismos. Todos os valores obtidos com os cortes se mantiveram na faixa de 3,20 a 3,40, valores mais elevados aos verificados por Canossa et al. (2014) em sucos elaborados com as cultivares Concord, Isabel Precoce e Bordô. Estes autores obtiveram resultados que variaram de 2,93 a 3,16. Dutra et al. (2014) encontraram média de 3,09 para sucos da cultivar BRS Cora (Silva et al., 2011) 3,30 para sucos de BRS Violeta.

Tabela 3. Resultados das análises químicas de sucos de uva preparados com uva Isabel e suas misturas com outras variedades produzidas no Vale do Rio do Peixe - SC, 2018.

\begin{tabular}{ccccc}
\hline & \multicolumn{4}{c}{ Variáveis } \\
\cline { 2 - 5 } Tratamentos & $\mathbf{p H}$ & $\begin{array}{c}\text { Acidez titulável } \\
\left(\mathbf{m E q} \mathbf{L}^{-1}\right)\end{array}$ & Sólidos Solúveis ('Brix) & Relação SS/AT \\
\hline ISA $^{* *}$ & $3,26 \mathrm{~d}^{*}$ & $103,50 \mathrm{c}$ & $17,63 \mathrm{c}$ & $22,71 \mathrm{c}$ \\
\hline ISA+RUB & $3,28 \mathrm{~cd}$ & $99,20 \mathrm{~cd}$ & $17,87 \mathrm{bc}$ & $24,02 \mathrm{abc}$ \\
\hline ISA+COR & $3,28 \mathrm{c}$ & $100,97 \mathrm{~cd}$ & $17,73 \mathrm{c}$ & $23,43 \mathrm{bc}$ \\
\hline ISA+VIO & $3,36 \mathrm{a}$ & $101,53 \mathrm{~cd}$ & $18,70 \mathrm{a}$ & $24,56 \mathrm{ab}$ \\
\hline ISA+CAR & $3,32 \mathrm{~b}$ & $96,10 \mathrm{~d}$ & $18,33 \mathrm{ab}$ & $25,44 \mathrm{a}$ \\
\hline ISA+MAG & $3,21 \mathrm{f}$ & $120,40 \mathrm{a}$ & $17,80 \mathrm{bc}$ & $19,72 \mathrm{~d}$ \\
\hline ISA+BOR & $3,23 \mathrm{e}$ & $110,50 \mathrm{~b}$ & $16,83 \mathrm{~d}$ & $20,32 \mathrm{~d}$ \\
\hline
\end{tabular}

*Valores seguidos pela mesma letra minúscula na coluna não diferiram estatisticamente pelo teste de Tukey a 5\% de probabilidade de erro. **ISA (Isabel); RUB (BRS Rúbea); COR (BRS Cora); VIO (BRS Violeta); CAR (BRS Carmem); MAG (BRS Magna) e BOR (Bordô). SS (sólidos solúveis) e AT (acidez titulável).

A maior acidez titulável foi verificada nos sucos de ISA+MAG, seguidos de ISA+BOR, apresentando diferença significativa entre si e em relação aos demais tratamentos. Os sucos de ISA+CAR obtiveram menor acidez dentre os tratamentos. O corte dos sucos das cultivares BRS Cora, BRS Violeta, BRS Rúbea e BRS Carmem ao suco de Isabel contribuíram para uma pequena redução da acidez titulável, enquanto os sucos de BRS Magna e Bordô apresentaram influência significativa no aumento desta variável. Mota et al. (2018) realizaram cortes de sucos nas proporções de $50 \%$ das cultivares BRS Violeta, Bordô e Concord com Isabel Precoce. Os autores alegaram que o corte de sucos de Isabel Precoce reduziu a acidez titulável dos sucos das demais variedades. Neste mesmo trabalho os autores avaliaram a qualidade de sucos varietais de diferentes cultivares, verificando que os sucos de BRS Carmem apresentaram o menor teor de acidez, enquanto os sucos de Bordô obtiveram o maior valor para esta variável. Resultados semelhantes foram verificados nos cortes com essas cultivares, no presente estudo. Silva et al. (2011) encontraram teores de acidez mais elevados para 
sucos de BRS Violeta em relação aos de Isabel, com valores de $110,66 \mathrm{mEq} \mathrm{L} \mathrm{L}^{-1}$ e $92,3 \mathrm{mEq} \mathrm{L}^{-1}$, respectivamente.

Os sucos oriundos do tratamento ISA+ VIO obtiveram a maior média para o teor de sólidos solúveis, não diferindo apenas do tratamento ISA+CAR. Os sucos de ISA+BOR obtiveram a menor média para esta variável diferindo dos demais tratamentos. No entanto, todos respeitaram o limite mínimo exigido em lei, que é de $14^{\circ}$ Brix (Brasil, 2018). De acordo com Chiarotti et al. (2011), a uva Bordô produzida nas regiões Sul e Sudeste apresentou deficiência na maturação, prejudicando o teor de sólidos solúveis das bagas, refletindo no suco. Em contrapartida, as novas cultivares lançadas apresentam maior potencial de acúmulo de sólidos solúveis (Camargo et al., 2010), o que pode ser evidenciado especialmente nas amostras que receberam suco de BRS Violeta e BRS Carmem.

O suco de uva possui teores de açúcar e acidez elevados, o que proporciona um equilíbrio entre os gostos doce e ácido (Gurak et al., 2012). Burin et al. (2010) alegam que a relação SS/AT indica a qualidade do suco e os valores para esta relação devem estar situados entre 15 e 45 . Choudhury (2001) considera que o valor desejável para este equilíbrio seja igual ou maior que 20. A maioria dos tratamentos, com exceção de ISA+MAG, apresentaram média superior ao valor citado por Choudhury (2001), sendo que os sucos de ISA+CAR obtiveram o maior resultado devido ao valor elevado de sólidos solúveis e menor teor de acidez. Comportamento oposto foi encontrado nos sucos de ISA+MAG, cuja acidez foi mais elevada e sólidos solúveis inferiores. A adição dos sucos das cultivares BRS Violeta, BRS Carmem, BRS Rúbea e BRS Cora ao suco 100\% Isabel elevaram os valores para esta variável, indicando maior equilíbrio gustativo. A mistura dos sucos de Isabel com os sucos das variedades citadas acima demonstra uma alternativa para melhorar a relação SS/AT em anos que as uvas não atinjam uma boa qualidade para processamento, devido ao período de maturação da cultivar Isabel nos estados do Sul ocorrer em períodos chuvosos, prejudicando o acúmulo de açúcares e degradação de ácidos, bem como o equilíbrio destes componentes no suco (Camargo et al., 2010). Lima et al. (2014) avaliaram cortes de $80 \%$ Isabel Precoce com $20 \%$ de BRS Violeta e $80 \%$ Isabel Precoce com 20\% de BRS Cora e encontraram valores de 25,8 e 20,7 para a relação SS/AT, respectivamente. Esses autores descrevem que a mistura de uvas com diferentes concentrações de sólidos solúveis e acidez total pode ser vantajosa para obtenção de sucos padronizados em relação a esses parâmetros, influenciando nas características gustativas da bebida.

As variáveis referentes à coloração dos sucos estão descritas na Tabela 4. A luminosidade da amostra é dada pela coordenada $* \mathrm{~L}$ que varia do branco $(* \mathrm{~L}=100)$ ao preto $\left({ }^{*} \mathrm{~L}=0\right)$, demonstrando que quanto menor for a leitura, mais escura encontra-se a amostra. De maneira geral, todas as variedades testadas nos cortes com Isabel demonstraram decréscimos nos valores da luminosidade, uma vez que o suco com 100\% Isabel diferiu dos demais em função do maior valor, ou seja, coloração inferior. A menor luminosidade foi observada nos sucos do tratamento ISA+VIO, seguido pelo tratamento ISA+CAR. O aumento da coloração dos sucos após os cortes pode ser reforçado pelos resultados da variação total de cor $\Delta \mathrm{E}^{*}$, utilizando o suco do tratamento ISA como padrão. Os resultados demonstram que as amostras que receberam o suco de BRS Violeta, seguidos por BRS Carmem, apresentaram maior variação de cor. Silva et al. (2011) avaliaram o índice de polifenóis totais e índice de cor de sucos de BRS Violeta e Isabel Precoce, encontrando uma diferença significativa entre as cultivares, alegando que a cultivar BRS Violeta possui uma expressiva capacidade de inferir cor nos sucos sendo uma excelente opção de cortes com sucos de outras variedades deficientes de coloração. Lima et al. (2014) também observaram aumento de cor do suco de Isabel Precoce quando misturado com suco de BRS Violeta. Alguns autores recomendam o uso das cultivares BRS Cora e BRS Violeta em cortes para melhoria da cor em proporções de 15 a $20 \%$ na formulação do suco (Lima et al., 2014). Ritschel et al. (2012) e Lima et al. (2014) fazem referência a cultivar BRS Magna, como uma alternativa para melhoria da cor, além das suas características favoráveis de adaptabilidade e aroma típico de Vitis labrusca. 
Tabela 4. Resultados das análises colorimétricas de sucos de uva preparados com uva Isabel e suas misturas com outras variedades produzidas no Vale do Rio do Peixe - SC, 2018.

\begin{tabular}{|c|c|c|c|c|c|c|}
\hline \multirow[b]{2}{*}{ Tratamentos } & \multicolumn{6}{|c|}{ Variáveis } \\
\hline & $\begin{array}{c}\text { Luminosidade } \\
\qquad\left(\mathrm{L}^{*}\right)\end{array}$ & $a^{*}$ & $\mathbf{b}^{*}$ & $\begin{array}{c}\text { Variação } \\
\text { total de cor } \\
\left(\Delta \mathbf{E}^{*}\right)\end{array}$ & $\begin{array}{c}\text { Tonalidade } \\
\text { da cor } \\
\left({ }^{\circ} \text { HUE }\right)\end{array}$ & $\begin{array}{c}\text { Intensidade } \\
\text { de cor } \\
\text { (Croma) }\end{array}$ \\
\hline ISA $^{* *}$ & $62,40 a^{*}$ & $30,54 \mathrm{f}$ & $6.65 \mathrm{c}$ & - & $12,40 \mathrm{~b}$ & $31,37 \mathrm{e}$ \\
\hline ISA+RUB & $57,20 \mathrm{~b}$ & $37,20 \mathrm{e}$ & $7.52 b c$ & $8,60 \mathrm{e}$ & $11,40 \mathrm{~b}$ & $37,92 \mathrm{~d}$ \\
\hline $\mathrm{ISA}+\mathrm{COR}$ & $53,53 \mathrm{c}$ & $38,20 \mathrm{de}$ & $8.32 \mathrm{bc}$ & $11,86 \mathrm{~d}$ & $12,30 \mathrm{~b}$ & $39,05 \mathrm{~d}$ \\
\hline $\mathrm{ISA}+\mathrm{VIO}$ & $35,23 \mathrm{e}$ & $48,31 \mathrm{a}$ & $18.18 \mathrm{a}$ & $34,50 \mathrm{a}$ & $20,70 \mathrm{a}$ & $51,63 \mathrm{a}$ \\
\hline ISA+CAR & $47,36 \mathrm{~d}$ & $43,93 \mathrm{~b}$ & $2.78 \mathrm{~d}$ & $20,53 \mathrm{~b}$ & $3,60 \mathrm{c}$ & $44,03 \mathrm{~b}$ \\
\hline $\mathrm{ISA}+\mathrm{MAG}$ & $51,61 \mathrm{c}$ & $40,58 \mathrm{~cd}$ & $9.26 \mathrm{~b}$ & $15 \mathrm{c}$ & $12,90 \mathrm{~b}$ & $41,64 \mathrm{c}$ \\
\hline ISA+BOR & $51,41 \mathrm{c}$ & $41,55 \mathrm{bc}$ & $3.96 \mathrm{~d}$ & $15,9 \mathrm{c}$ & $5,40 \mathrm{c}$ & $41,77 \mathrm{c}$ \\
\hline
\end{tabular}

*Valores seguidos pela mesma letra minúscula na coluna não diferiram estatisticamente pelo teste de Tukey a $5 \%$ de probabilidade de erro. **ISA (Isabel); RUB (BRS Rúbea); COR (BRS Cora); VIO (BRS Violeta); CAR (BRS Carmem); MAG (BRS Magna) e BOR (Bordô).

As respostas de cor $\mathrm{a}^{*} \mathrm{e} \mathrm{b}^{*}$ indicam as direções das cores: +a para o vermelho, -a para o verde, $+\mathrm{b}$ em direção ao amarelo e -b, ao azul. À medida que o valor destas coordenadas aumenta, o ponto se distância do centro e eleva a saturação da cor (Gurak et al. 2012). Dentre as coordenadas a* e b*, os maiores valores foram verificados no tratamento ISA+VIO diferindo dos demais. Essa mesma diferença persistiu para as variáveis de tonalidade e intensidade de cor. Apesar da maior intensidade de cor inferida pelos sucos de BRS Violeta, observa-se uma tendência de coloração com nuances amarelados, uma vez que a tonalidade dada pelo ângulo ${ }^{\circ} \mathrm{HUE}$ indica uma coloração na faixa do vermelho ao laranja avermelhado, de acordo com as escala CIELAB, o que pode indicar uma possível oxidação.

Esses resultados podem ocorrer devido ao alto índice de polifenóis encontrados nos sucos de BRS Violeta que podem sofrer oxidações e que transformam a estrutura e a cor das diferentes moléculas, podendo ser por antocianinas, que são particularmente degradadas em parte na cor vermelha ou taninos que se polimerizam e escurecem (Ferri et al., 2017). Os sucos dos demais tratamentos se mantiveram na faixa do vermelho púrpura, faixa considerada ideal para Pereira et al. (2008). Estes autores avaliaram sensorialmente sucos de Concord e Bordô, sendo que os sucos de Concord, julgados como vermelho tijolo, foram considerados insatisfatórios, enquanto os sucos de Bordô foram classificados como vermelho violáceo e melhor aceitos.

Ferri et al. (2017) avaliaram cortes de sucos de Bordô com Isabel em diferentes proporções, verificando que globalmente a tonalidade diminuiu à medida que o percentual de uva Isabel aumentou. Lima et al. (2014) obtiveram diferenças significativas na intensidade da cor nos cortes de Isabel Precoce com BRS Cora e BRS Violeta em relação a sucos de Isabel Precoce 100\%.

Os atributos analisados na avaliação sensorial dos sucos estão listados na Tabela 5. Foram observadas diferenças significativas apenas para cor e acidez. Os sucos de ISA + VIO, ISA+CAR e ISA+BOR receberam as maiores notas para o atributo cor diferindo de ISA, ISA+RUB e ISA+COR. Os sucos de ISA não diferiram apenas de ISA+RUB. A cor está relacionada à composição fenólica e é o atributo mais importante, usado com outras variáveis para indicar a qualidade do suco de uva e influenciar os consumidores no momento da compra (Burin et al., 2010; Mota et al., 2018). Nos sucos avaliados sensorialmente por Borges et al. (2011) houve diferença significativa para cor, em que as maiores médias foram para as amostras de sucos da cultivar Isabel em cortes com BRS Rúbea, BRS Cora, BRS Carmem e BRS Violeta. A amostra elaborada somente com a Isabel e a combinada com Concord obtiveram médias menores de aceitação pelo grupo de provadores. Os autores justificam que estes resultados demonstram que a maior pigmentação do suco, obtida pelos cortes 
com materiais oriundos de melhoramento genético, foi melhor aceita pelos julgadores quando comparada com a coloração dos sucos tradicionais, como o suco puro de Isabel ou sua combinação com Concord. Mota et al. (2018) verificaram que os sucos oriundos de cortes foram preferidos pelos avaliadores em relação aos sucos varietais, sendo que a mistura de BRS Violeta com Isabel Precoce obteve maior preferência por parte dos avaliadores. Os autores acreditam que este resultado foi devido ao maior conteúdo de polifenóis e consequentemente maior coloração. Canossa et al. (2014) trabalharam com sucos de Isabel Precoce, Concord e Bordô, verificando que os atributos sensoriais avaliados foram liderados pela cultivar Bordô, que apresentou a maior intensidade de coloração e as maiores notas de aroma, sabor e corpo. Esse resultado demonstra que um suco de uva com maior intensidade de coloração, potencial aromático e presença de corpo e sabor é mais atrativo ao consumidor.

Tabela 5. Resultados das análises sensoriais de sucos de uva preparados com uva Isabel e suas misturas com outras variedades produzidas no Vale do Rio do Peixe - SC, 2018.

\begin{tabular}{cccccc}
\hline & \multicolumn{3}{c}{ Atributos } \\
\cline { 2 - 5 } Tratamentos & Cor & Aroma & Acidez & Doçura & $\begin{array}{c}\text { Equilíbrio } \\
\text { açúcar/acidez }\end{array}$ \\
\hline ISA $^{* * *}$ & $6,25 \mathrm{c}^{*}$ & $6,7 \mathrm{~ns}^{* *}$ & $5,40 \mathrm{c}$ & $7,60 \mathrm{~ns}$ & $6,55 \mathrm{~ns}$ \\
\hline ISA+RUB & $6,80 \mathrm{bc}$ & 7,00 & $5,60 \mathrm{bc}$ & 7,90 & 6,55 \\
\hline ISA+COR & $7,05 \mathrm{~b}$ & 7,15 & $6,00 \mathrm{abc}$ & 7,45 & 6,86 \\
\hline ISA+VIO & $7,95 \mathrm{a}$ & 7,05 & $5,60 \mathrm{bc}$ & 7,30 & 7,05 \\
\hline ISA+CAR & $7,90 \mathrm{a}$ & 6,90 & $5,30 \mathrm{c}$ & 7,75 & 6,50 \\
\hline ISA+MAG & $7,40 \mathrm{ab}$ & 7,10 & $7,30 \mathrm{a}$ & 6,65 & 6,75 \\
\hline ISA+BOR & $7,70 \mathrm{a}$ & 6,95 & $7,00 \mathrm{ab}$ & 6,65 & 6,80 \\
\hline
\end{tabular}

*Valores seguidos pela mesma letra minúscula na coluna não diferiram estatisticamente pelo teste de Tukey a $5 \%$ de probabilidade de erro. **ns- não significativo pelo teste de Tukey a 5\% de probabilidade de erro. ***ISA (Isabel); RUB (BRS Rúbea); COR (BRS Cora); VIO (BRS Violeta); CAR (BRS Carmem); MAG (BRS Magna) e BOR (Bordô).

Os julgadores perceberam a maior acidez nos sucos de ISA+MAG e ISA+BOR, não diferindo apenas de ISA $+\mathrm{COR}$, indo ao encontro dos resultados das análises químicas. A variável doçura bem como seu equilíbrio com a acidez não apresentaram diferenças significativas entre os tratamentos. No entanto, é possível observar que os sucos apresentaram uma doçura maior em relação acidez, mantendo o equilíbrio entre estes atributos, uma vez que as notas se mantiveram todas acima de 6 (levando em conta a escala de 0 a 9 pontos, considera-se que quanto mais próximo de 9 , mais perceptíveis são os aspectos sensoriais). O sabor doce reflete e colabora com a estrutura, juntamente com os taninos, enquanto a acidez participa do frescor e conservação do produto, sendo o equilíbrio destes que garante a qualidade da bebida.

Menezzes et al. (2011) relatam que assim como as sensações gustativas são determinantes na aceitação dos sucos, as variáveis olfativas são igualmente importantes, uma vez que estas são o segundo fator a ser avaliado durante a análise sensorial. Os sucos de uva são conhecidos pelo aroma característico das uvas Vitis labrusca, sendo que os compostos responsáveis por essa variável são ésteres voláteis, principalmente antranilato de metila, conhecido como aroma foxado. Os resultados para a variável aroma não apresentaram diferenças significativas entre as amostras testadas e mantiveram as notas em torno de 7 , demonstrando maior intensidade, uma vez que as notas foram mais próximas do escore "muito agradável" da escala utilizada. Pereira et al. (2008) avaliaram sensorialmente sucos de cinco cultivares, sendo que os sucos de Isabel, Bordô e BRS Rúbea mostraram-se mais tipicamente relacionados com o aroma foxado. 
Os resultados da análise sensorial demonstram que a adição de $30 \%$ de sucos de diferentes cultivares ao suco de Isabel não foi suficiente para alterar significativamente os atributos de aroma, doçura e equilíbrio açúcar/acidez. No entanto, todas as cultivares contribuíram para o incremento da coloração, fator benéfico para a qualidade do suco. Borges et al. (2011) alertam quanto à necessidade de cautela na mudança da matriz varietal dos sucos brasileiros, uma vez que o paladar do consumidor está habituado as características dos sucos de Isabel.

\section{Conclusão}

A adição dos sucos das cultivares BRS Carmem, BRS Magna e Bordô bem como BRS Violeta e BRS Carmem alteraram a acidez total e sólidos solúveis totais, respectivamente, quando comparados os cortes com o suco varietal de Isabel.

Todas as cultivares testadas influenciaram as características colorimétricas do suco, mostrando-se alternativas para melhoria da cor de sucos de Isabel.

Sensorialmente os atributos de coloração e acidez foram influenciados pelos diferentes cortes.

\section{Referências}

Associação Brasileira De Normas Técnicas - ABNT (2014). Análise sensorial - Metodologia - Orientações gerais (NBR ISO 6658:2014). São Paulo: ABNT.

Bender, A., Malgarim, M. B., Costa, V. B., Silva, R. S., Guadagnin, V., \& Andrade, S. B. (2017). Influência de variedades de uvas Vitis vinifera nas características físico-químicas e na aceitação sensorial do suco. Ciência e Técnica Vitivinícola, 32(6), 213-230.

Borges, R. S., Prudêncio, S. H., Roberto, S. R., \& Assis, A. M. (2011). Avaliação sensorial de suco de uva cv. Isabel em cortes com diferentes cultivares. Revista Brasileira de Fruticultura, 33(spe1), 584-591. http://dx.doi.org/10.1590/S010029452011000500080

Brasil. Ministério da Saúde. Secretaria de Vigilância Sanitária. (2018, março 9). Complementação dos padrões de identidade e qualidade do vinho e dos derivados da uva e do vinho (Instrução Normativa n 14, de 08 de fevereiro de 2018). Diário Oficial [da] República Federativa do Brasil, Brasília, Seção 1, p. 4-6.

Burin, V. M., Falcão, L. D., Gonzaga, L. V., Fett, R., Rosier, J. P., \& Bordignon-Luiz, M. T. (2010). Colour, phenolic content and antioxidant activity of grape juice. Food Science and Technology (Campinas), 4(30), 1027-1032. http://dx.doi.org/10.1590/S0101-20612010000400030

Camargo, U. A., Maia, J. D. G., \& Ritschel, P. S. (2010). Novas cultivares de uva (64 p.). Bento Gonçalves. Embrapa Uva e Vinho.

Canossa S., Mossmann, D. L., Dachery, B., \& Manfroi, V. (2014). Avaliação físico-química dos sucos das uvas provenientes da espécie de Vitis labrusca acondicionados em diferentes recipientes. Revista Brasileira de Viticultura e Enologia, 6, 66-71.

Castilhos, M. B. M., Garcia Maia, J. D., Gómez-Alonso, S., Del Bianchi, V. L., \& Hermosín-Gutiérrez, I. (2016). Sensory acceptance drivers of pre-fermentation dehydration and submerged cap red wines produced from Vitis labrusca hybrid grapes. Lebensmittel-Wissenschaft + Technologie, 69, 82-90. http://dx.doi.org/10.1016/j.Iwt.2016.01.043

Chiarotti, F., Guerios, I. T., Cuquel, F. L., \& Biasi, L. A. (2011). Melhoria da qualidade de uva 'Bordô' para produção de vinho e suco de uva. Revista Brasileira de Fruticultura, 33(spe1), 618-624. http://dx.doi.org/10.1590/S0100-29452011000500085

Choudhury, M. M. (Ed.). (2001). Uva de mesa: pós-colheita (Vol. 12, 55 p., Série Frutas do Brasil). Brasília: Embrapa Informação Tecnológica; Petrolina-PE: Embrapa Semiárido.

Dutra, M. da C. P., Lima, M. S., Barros, A. P. A., Mascarenhas, R. J., \& Lafisca, A. (2014). Influência da variedade de uvas nas características analíticas e aceitação sensorial do suco artesanal. Revista Brasileira de Produtos Agroindustriais, 16(3), 265272. http://dx.doi.org/10.15871/1517-8595/rbpa.v16n3p265-272

Empresa de Pesquisa e Extensão Rural de Santa Catarina - EPAGRI. (2018). Síntese Anual da Agricultura de Santa Catarina 2017-2018. Recuperado em 8 de setembro de 2018, de http://webdoc.epagri.sc.gov.br/sintese.pdf

Ferri, V. C., Sainz, R. L., \& Bandeira, P. S. (2017). Aceitação de blends de uvas 'Bordô' e 'Isabel' em sucos. Brazilian Journal of Food Research, 8(3), 88-101. http://dx.doi.org/10.3895/rebrapa.v8n3.3667

Fongaro, C., Cavagnolli, N. I., \& Spada, P. K. W. D. S. (2016). Evaluation of physicochemical parameters of grape juices produced in the Serra Gaúcha. In Proceedings of the 39th BIO Web of Conferences (Vol 7). France: EDP Sciences - Web of Conferences. http://dx.doi.org/10.1051/bioconf/20160701008

Gurak, P. D., Silva, M. C., Matta, V. M., Rocha-Leão, M. H., \& Cabra, L. M. C. (2012). Avaliação de parâmetros físico-químicos de sucos de uva integral, néctares de uva e néctares de uva light. Revista de Ciências Exatas, 27-31(1), 7-22. 
International Organisation of Vine and Wine - OIV. (2009). Compendium of international methods of wine and must analysis. Paris: International Organisation of Vine and Wine.

Lima, M. S., Silani, I. S. V., Toaldo, I. M., Corrêa, L. C., Biasoto, A. C. T., Pereira, G. E., Bordignon-Luiz, M. T., \& Ninow, J. L. (2014). Phenolic compounds, organic acids and antioxidant activity of grape juices produced from new Brazilian varieties planted in the Northeast Region of Brazil. Food Chemistry, 161, 94-103. PMid:24837926. http://dx.doi.org/10.1016/j.foodchem.2014.03.109

McGuire, R. G. (1992). Reporting of objective color measurements. HortScience, 27(12), 1254-1555. http://dx.doi.org/10.21273/HORTSCI.27.12.1254

Mello, L. M. R., \& Machado, C. A. E. (2018). Dados da viticultura. Bento Gonçalves: EMBRAPA. Recuperado em 8 de setembro de 2018, de http://vitibrasil.cnpuv.embrapa.br/

Menezzes, F., Messias, G. M., \& Barros, N. E. F. (2011). Análise sensorial de suco de uva orgânico - Teste de aceitação. Revista Eletrônica Novo Enfoque, 12(12), 1.

Miotto, L. C. V., Mota, R. V., Souza, C. R., França, D. V. C., Dias, F. A. N., Pimentel, R. M. A., Dal'Osto, M. C., \& Regina, M. A. (2014). Agronomic evaluation of 'Bordô' grapevine (Ives) clones. Scientia Agrícola, 71(6), 458-463. http://dx.doi.org/10.1590/0103-9016-2013-0388

Mota, R. V., Glória, M. B. A., Souza, B. S., Peregrino, I., Pimentel, R. M. A., Dias, F. A. N., Souza, L. C., Souza, A. L., \& Regina, M. A. (2018). Bioactive compounds and juice quality from selected grape cultivars. Bragantia, 77(1), 62-73. http://dx.doi.org/10.1590/1678-4499.2016369

Pereira, G. E., Lima, L. C. O., Regina, M. A., Rosier, J.-P., Ferraz, V., \& Mourão Junior, M. (2008). Avaliação do potencial de cinco cultivares devideiras americanas para sucos de uva no sul de minas gerais. Ciência e Agrotecnologia, 32(5), 1531-1537. http://dx.doi.org/10.1590/S1413-70542008000500026

Ritschel, P., Maia, J. D. G., Camargo, U. A., Zanus, M. C., Souza, R. T., \& Fajardo, T. G. M. (2012). 'BRS Magna' nova cultivar de uva para suco com ampla adaptação climática (Vol. 125, Comunicado Técnico). Brasília: Embrapa Informação Tecnológica. Rizzon, L. A., \& Meneguzzo, J. (2007). Suco de uva (45 p.). Brasília: Embrapa Informação Tecnológica.

Silva, G. G., Nascimento, R. L., Oliveira, V. S., Araújo, A. J. B., Oliveira, J. B., \& Pereira, G. E. (2011). Características físicoquímicas de sucos de uvas 'Isabel Precoce' e 'BRS Violeta' elaborados no Nordeste do Brasil. In Anais da $6^{a}$ Jornada de Iniciação Científica da Embrapa Semiárido (pp. 353-359). Petrolina: Embrapa Semiárido

Toaldo, I. M., Cruz, F. A., Alves, T. L., Gois, J. S., Borges, D. L. G., Cunha, H. P., Silva, E. L., \& Bordignon-Luiz, M. T. (2015). Bioactive potential of Vitis labrusca L. grape juices from the Southern Region of Brazil: phenolic and elemental composition and effect on lipid peroxidation in healthy subjects. Food Chemistry, 173, 527-535. PMid:25466055. http://dx.doi.org/10.1016/j.foodchem.2014.09.171

Toaldo, I. M., Fogolari, O., Pimentel, G. C., Gois, J. S., Borges, D. L. G., Caliari, V., \& Bordignon-Luiz, M. (2013). Effect of grape seeds on the polyphenol bioactive content and elemental composition by ICP-MS of grape juices from Vitis labrusca $\mathrm{L}$. Lebensmittel-Wissenschaft + Technologie, 53(1), 1-8. http://dx.doi.org/10.1016/j.Iwt.2013.02.028 